\title{
Validación operacional de un ELISA comercial para Brucella ovis, Chile
}

\author{
Operational validation of a commercial ELISA for Brucella ovis, Chile \\ J F Alvarez ${ }^{\text {a* }}$, R Veneros ${ }^{\mathrm{b}}$, O González \\ aServicio Agrícola y Ganadero, Sector Ultima Esperanza, Puerto Natales, Chile.

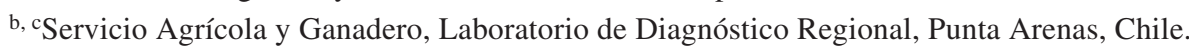

\section{SUMMARY}

\begin{abstract}
Sensitivity and specificity are usually estimated for a reference population, and these differ from the operational interpretation. The aim of this study was to carry out an operational validation of a commercial Enzyme-Linked Immunosorbent Assay (ELISA) for Brucella ovis in the sheep population of Magallanes Region. The assay was validated using sera from 82 rams with bacterial isolation and 421 sera from rams with culturenegative semen and not reacting to agar gel immunodiffusion test. The cut-off point was $58.15 \%$ optical density, estimated by using the receiveroperating characteristic method; with $82.9 \%$ of sensibility and $91.4 \%$ of specificity; the low sensibility indicates the failure of the ELISA to detect a great number of positives, therefore an additional diagnostic test must be used in eradication programmes; the low positive predictive value was mainly influenced by the low prevalence. The estimated parameters should be used in the interpretation of future diagnostic tests and an operational validation must be carried out when a new diagnostic test is adopted, establishing the parameters under which the laboratory will work, as a way to minimize the misdiagnoses.
\end{abstract}

Palabras clave: validación operacional, ELISA, Brucella ovis.

Key words: validation operational, ELISA, Brucella ovis.

\section{INTRODUCCION}

La Región de Magallanes y Antártica Chilena posee una superficie de uso agropecuario cercana a 3,5 millones de hectáreas, concentra el $52 \%$ de la masa ovina de Chile, con una dotación aproximada de 1.923.694 cabezas (Chile 1996), las que en un $97 \%$ corresponden a la raza Corriedale. Esta Región es una zona geográficamente aislada, sin comunicación directa con las demás regiones de Chile; posee una producción extensiva y cuenta con un nivel zoosanitario de excelencia, siendo una Región libre de Sarna Ovina (Psoroptes ovis), Fiebre "Q" (Coxiella burnetii) y provisionalmente libre de Aborto Enzoótico Ovino (Chlamydophila abortus), condición debida a sus características insulares y por la escasa introducción de nuevo material genético a la población (Rowland y col 2003); pese a esto y al igual que en otros lugares con ganadería ovina, la producción de carne, lana, cueros y leche se encuentra afectada por patologías reproductivas como la "epididimitis contagiosa del carnero" (Arévalo y col 2004). Dicha patología es causada por la bacteria Brucella ovis (B. ovis), se caracteriza por ser de transmisión venérea y por presentar clínicamente,

Aceptado: 19.12.2006.

* I. Carrera Pinto N 566, Puerto Natales, Chile; francisco.alvarez@ sag.gob.cl en los carneros afectados, epididimitis crónica, vesiculitis seminal, fertilidad disminuida, anomalías seminales (Paolicchi y col 1992) y atrofia de los testículos (Martin y Aitken 2000). En la hembra puede ocasionar infertilidad transitoria, mortalidad embrionaria y nacimiento de corderos débiles (Paolicchi y col 1992). Fue descrita por primera vez en Nueva Zelanda y Australia en 1942 (Reichel y col 1994), posteriormente ha sido reportada en Africa, algunos países europeos y América (Gaffuri y Garbarino 1999).

Las pérdidas económicas se producen fundamentalmente por la disminución de los porcentajes de parición y señalada (Paolicchi y col 1992), existiendo también pérdidas indirectas debidas al acortamiento de la vida reproductiva con la consiguiente eliminación de los reproductores, un menor valor de venta y un aumento del porcentaje de reposición de machos, mayor número de carneros para utilizar en el servicio y establecimiento de inspecciones preencaste (Paolicchi 2002 ${ }^{1}$ ).

El control de esta enfermedad se basa en el principio de la identificación y la eliminación de los animales seropositivos y/o con epididimitis clínica, evitando la introducción de carneros infectados (Estein 1999). Por

Comunicación personal. Fernando Paolicchi. Estudios efectuados sobre brucelosis ovina (Epididimitis de los carneros por Brucella ovis). EEA INTA - Facultad de Ciencias Agrarias, UNMdP, Grupo de Sanidad Animal, Unidad Integrada Balcarce, Argentina. 
este motivo han utilizado diferentes pruebas de variada naturaleza, destacando en primer lugar, como método directo de diagnóstico, el cultivo microbiológico de semen, el que se realiza en un medio selectivo y a una atmósfera de 5 a $10 \%$ de $\mathrm{CO}_{2}$, debiendo ser observado durante 10 días antes de dar un cultivo como negativo, aunque este resultado no descarta la presencia de $B$. ovis, ya que la naturaleza crónica de la enfermedad conlleva una liberación intermitente del germen (Estein 1999); además su eficiencia depende de microorganismos contaminantes y el tiempo entre la toma de la muestra y la siembra (Paolicchi 1992). La palpación testicular de los carneros es utilizada como un método diagnóstico simple y rápido, pero tiene una baja especificidad, un $50 \%$ de los animales infectados no desarrolla una epididimitis palpable (Blasco 1990), siendo portadores y transmisores de la enfermedad (Kott y col 1988); además es necesario tener en cuenta la presencia de otras bacterias que causan epididimitis clínica (Zamora y col 1977). Por otro lado, se encuentran las pruebas serológicas, entre las que destaca la fijación del complemento como prueba de referencia a nivel internacional (http://www.oie.int/fr/ normes/mmanual/A_00068.http) y prueba oficial en Chile (http://www2.sag.gob.cl/Pecuaria/exigencias_ sanitarias_especificas/word/Ovinos_Reproduccion_ Resoluc_1995_97.DOC), teniendo la desventaja de ser compleja, ya que requiere de una rigurosa estandarización y titulación de cada uno de los reactivos (Rahaley y col 1984), dependiendo los resultados de la temperatura a la cual se desarrolle, no siendo compatible con sueros hemolizados o anticomplementarios (Searson 1982), y presentando además una menor sensibilidad y especificidad que el test de ELISA (Enzyme-Linked Immunosorbent Assay) (Estein 1999); esta última técnica posee además las ventajas de permitir el procesamiento de gran número de muestras simultáneamente y de que la hemólisis o anticomplementariedad del suero no afectan la reacción (Vigliocco y col 1997).

La especificidad y sensibilidad para un determinado test diagnóstico están estimadas para una población de referencia, con una distribución característica de cofactores, entre los que destacan las condiciones del laboratorio, test de oro y puntos de corte. Estos factores difieren en el desarrollo e interpretación de una prueba a nivel operacional o de terreno, ya que se aplican en poblaciones no homogéneas (Greiner y Gardner 2000a).

El objetivo del presente trabajo es validar desde un punto de vista operacional un test de ELISA comercial para el diagnóstico de B. ovis (CHEKITBrucella ovis $^{2}$ ) en la población ovina de la Región de Magallanes, Chile.

\footnotetext{
IDEXX Laboratories B.V., Nederland.
}

\section{MATERIAL Y METODOS}

LUGAR DEL ESTUDIO, OBTENCION Y NATURALEZA DE LAS MUESTRAS

El estudio se realizó en la Región de Magallanes (48 $36^{\prime}$ a $56^{\circ} 30^{\prime}$ latidud sur, 66 $25^{\prime}$ a $75^{\circ} 40^{\prime}$ longitud oeste), Chile. Se obtuvieron muestras de carneros, consistentes en semen, mediante el uso de electroeyaculador y paralelamente suero sanguíneo; ambos materiales fueron remitidos al Laboratorio de Diagnóstico Regional del Servicio Agrícola y Ganadero, en la ciudad de Punta Arenas, para su posterior procesamiento y/o almacenaje.

\section{TAMAÑO MUESTRAL}

Para el cálculo del tamaño muestral se utilizó la siguiente fórmula (Greiner y Gardner 2000a):

$$
\mathrm{n}=\left(\left(\mathrm{z}_{1}-\alpha / 2\right) / \mathrm{e}\right)^{2} \times \operatorname{Se}(1-\mathrm{Se})
$$

En donde:

$z_{1}-\alpha / 2=1,96$ (95\% nivel de confianza).

Error $(e)=0,035$.

Sensibilidad estimada $(\mathrm{Se})=0,973$ (Ruelas y Rosadio 1999).

Se estimó un tamaño muestral de 82 sueros positivos, los cuales fueron seleccionados utilizando un diseño transversal con verificación parcial (Greiner y Gardner $2000^{\mathrm{a}}$ ), en un total de 35 explotaciones ovinas.

\section{SUEROS DE REFERENCIA}

Sueros positivos. Se emplearon 82 sueros sanguíneos obtenidos en terreno y pertenecientes a carneros con aislamiento de $B$. ovis en semen mediante cultivo microbiológico, de acuerdo a la técnica descrita por la Organización Mundial de Sanidad Animal (http:// www.oie.int/fr/normes/mmanual/A_00068.htm), y sin prueba serológica adicional.

Sueros negativos. Se utilizaron 421 sueros sanguíneos obtenidos en terreno, provenientes de carneros con cultivo de semen sin aislamiento de $B$. ovis e inmunodifusión en agar gel con resultado negativo, de acuerdo a las técnicas descritas por la Organización Mundial de Sanidad Animal (http://www.oie.int/fr/normes/mmanual/ A_00068.htm).

\section{ELISA INDIRECTO}

Se utilizó un lector ELISA modelo Multiscan $\mathrm{EX}^{3}$, kit ELISA indirecto (CHEKIT-Brucella ovis) que detecta anticuerpos a $B$. ovis en suero ovino; el desarrollo de la prueba se realizó según lo establecido por el fabricante

\footnotetext{
3 LABSYSTEM, Finland.
} 
(http://www.bommeli.com/checkit/chek_f_m.htm), de la misma forma los porcentajes de densidad óptica (\%DO) se estimaron utilizando los sueros controles aportados por el kit comercial y de acuerdo a la siguiente fórmula:

$\% \mathrm{DO}=\frac{\mathrm{DO} \text { de la muestra }-\mathrm{DO} \text { controles negativos }}{\mathrm{DO} \text { controles positivos }-\mathrm{DO} \text { controles negativos }} \times 100$

\section{ANALISIS ESTADISTICOS}

Los análisis estadísticos y la estimación del punto de corte se realizaron utilizando el Software MedCalc(C) Versión 8.0.2.04, específicamente mediante el método receiver-operating characteristic (ROC) (Zweig y Campbell 1993, Greiner y col 2000 ). Para el cálculo de los valores predictivos se empleó una prevalencia intrarrebaño de 7,96\% (Otzen $2000^{5}$ ) y se emplearon las siguientes fórmulas:

$$
\begin{aligned}
& \text { Valor predictivo positivo }=\frac{\mathrm{P} \times \mathrm{Se}}{\mathrm{P} \times \mathrm{Se}+(100-\mathrm{P}) \times(100-\mathrm{Esp})} \times 100 \\
& \text { Valor predictivo negativo }=\frac{(100-\mathrm{P}) \times \mathrm{Esp}}{(100-\mathrm{P}) \times \mathrm{Esp}+\mathrm{P} \times(100-\mathrm{Se})} \times 100
\end{aligned}
$$$$
\begin{aligned}
& \text { Sensibilidad }(\mathrm{Se})=\frac{\text { Sueros ELISA verdaderos positivos }}{\text { Sueros cultivo positivo }} \times 100 \\
& \text { Especificidad }(\text { Esp })=\frac{\text { Sueros ELISA verdaderos negativos }}{\text { Sueros cultivo negativo e Inmunodifusión en agar gel negativo }} \times 100
\end{aligned}
$$

En donde: $\mathrm{P}=$ Prevalencia.

\section{RESULTADOS Y DISCUSION}

El punto de corte estimado con el análisis ROC fue de un $58,15 \%$ DO, es decir, sobre este nivel de porcentaje de densidad óptica los sueros fueron considerados como positivos (figura 1). Este valor deberá utilizarse en los futuros análisis diagnósticos.

De acuerdo al punto de corte, anteriormente establecido, el ELISA comercial logró discriminar 68 de los 82 sueros positivos y 385 de los 421 negativos (cuadro 1), estimándose una sensibilidad del 82,9\% (95\% IC 73,0\% - $90.3 \%$ ) y una especificidad de $91,4 \%$ (95\% IC $88,4 \%$ $-93,9 \%)$; en tanto, el valor predictivo positivo fue de $45,6 \%$ y el negativo fue de $98,4 \%$.

Los valores de sensibilidad y especificidad estimados son considerablemente más bajos que lo reportado por otros autores (Worthington y col 1984, Walter y col

Frank Schoonjans, Belgium.

5 Comunicación personal. Informe muestreo serológico para Brucella ovis en la Provincia de Magallanes. Gerardo Otzen, Médico Veterinario, Jefe Sector Magallanes, SAG, XII Región de Magallanes y Antártica Chilena, Chile.

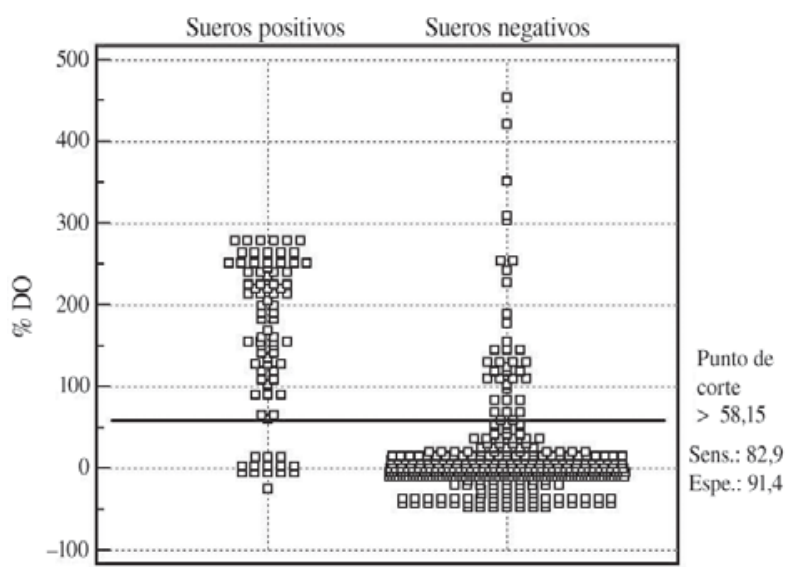

Figura 1. Diagrama de puntos de los porcentajes de densidad óptica del ELISA comercial para B. ovis. Servicio Agrícola y Ganadero, Chile.

ROC points diagram of the Optical Density percentages of the commercial ELISA for B. ovis. Agricultural and Livestock Service, Chile.

Cuadro 1. Resultados del ELISA comercial a B. ovis, según sueros de referencia. Servicio Agrícola y Ganadero, Chile.

Results of the B. ovis commercial ELISA, according to reference sera. Agricultural and Livestock Service, Chile.

\begin{tabular}{ccccc}
\hline & & \multicolumn{2}{c}{ Sueros de referencia } & Total \\
\cline { 3 - 4 } & & Positivos & Negativos & \\
\hline \multirow{2}{*}{ ELISA } & Positivo & 68 & 36 & 104 \\
& Negativo & 14 & 385 & 399 \\
\hline \multirow{2}{*}{} & Total & 82 & 421 & 503 \\
\hline
\end{tabular}

1985, Ruelas y Rosadio 1999), aunque ellos prepararon y utilizaron su propio ELISA. Estas diferencias pueden deberse al empleo de distintas estrategias de muestreo (Greiner y Gardner 2000ª) y además a las poblaciones de referencia usadas para los procesos de validación, considerando que el presente estudio se realizó en una población no homogénea, con cofactores como raza, edad y nivel sanitario específicos para la población ovina de la Región de Magallanes.

Cabe destacar que la baja sensibilidad obtenida, a pesar de utilizar la prueba de oro descrita para los animales positivos, el cultivo y aislamiento del agente en semen (Ruelas y Rosadio 1999), puede estar alterada por factores inherentes a la población, como por ejemplo el nivel de prevalencia de la enfermedad (Brenner y Gefeller 1997), específicamente factores relacionados con la infección, como el estado y severidad del cuadro clínico, este difiere en poblaciones con baja y alta prevalencia (Greiner y Gardner 2000ª), fenómeno que puede relacionarse con el nivel de anticuerpos detectables y debiera estudiarse con mayor detención. La sensibilidad anterior- 
mente estimada indicaría la incapacidad del ELISA comercial para detectar un número no despreciable de individuos positivos, es decir generaría una cantidad considerable de falsos negativos. Esto se traduciría a nivel de terreno en una mantención latente de la enfermedad a nivel predial (Alonso y col 1995), por lo que en etapas de erradicación se deberá utilizar una técnica diagnóstica adicional, ya sea en forma paralela o en serie, con resultados negativos en al menos dos periodos sucesivos. En este sentido los mejores resultados se han obtenido combinando inmunodifusión en agar gel y ELISA con sensibilidades del 100\% (Estein 1999); adicionalmente existen programas exitosos de erradicación en Australia y Nueva Zelandia, utilizando palpación testicular y la fijación del complemento (Niilo 1984); se debe destacar que, en los casos anteriormente nombrados, el costo se ve aumentado considerablemente al incluir una prueba adicional al esquema de erradicación.

Por otro lado, la especificidad estimada puede estar influenciada por la existencia de animales que al momento de la toma de la muestra de semen no estén excretando la bacteria (Estein 1999), y que posteriormente sean negativos a la prueba de inmunodifusión en agar gel, considerándolos como negativos. Este problema se podría minimizar realizando cultivos seriados de semen y/o pruebas seriadas de inmunodifusión en agar gel, aumentando la probabilidad de detectar individuos positivos a B. ovis.

El bajo valor predictivo positivo estaría condicionado, en su mayoría por la baja prevalencia utilizada para su estimación (Jacobson 1988).

Finalmente con los resultados obtenidos en el presente estudio se reafirma la necesidad de realizar los procesos de validación operacional cada vez que se adopte el uso de un nuevo ELISA comercial u otro test, para la detección de $B$. ovis, con el objetivo de establecer los parámetros con los cuales trabajará el laboratorio y de esta forma minimizar los errores de diagnóstico.

\section{RESUMEN}

La sensibilidad y especificidad diagnóstica se estiman para una población de referencia, estas difieren de la interpretación operacional o de terreno. El objetivo del presente trabajo es validar operacionalmente un ELISA (Enzyme-Linked Immunosorbent Assay) comercial para Brucella ovis en la población ovina de Magallanes. Se emplearon 82 sueros de carneros con aislamiento de B. ovis en semen y 421 sueros de animales con cultivo microbiológico de semen negativo e inmunodifusión en agar gel con resultado negativo. Se estableció un punto de corte de $58,15 \%$ de densidad óptica, estimado con el método receiver-operating characteristic; se estimó una sensibilidad de $82,9 \%$ y una especificidad de $91,4 \%$; la baja sensibilidad obtenida indica la incapacidad del ELISA para detectar un número considerable de positivos, por lo que en erradicación se deberá utilizar una técnica diagnóstica adicional; el bajo valor predictivo positivo fue influenciado en su mayoría por la baja prevalencia. Los parámetros estimados deberán utilizarse en la interpretación de los futuros análisis diagnósticos y se deberá realizar una validación operacional cuando se adopte una nueva técnica diagnóstica, estableciendo los parámetros con los cuales trabajará el laboratorio y de esta forma minimizar los errores de diagnóstico.

\section{AGRADECIMIENTOS}

A todos los colegas Médicos Veterinarios y Técnicos del Servicio Agrícola y Ganadero de la XII Región, quienes colaboraron en la obtención de las muestras, un reconocimiento muy especial por su importante aporte para la realización del presente estudio.

\section{REFERENCIAS}

Alonso O, X Rojas, E Guzmán. 1995. Uso de una técnica de ELISA indirecto para el diagnóstico de Brucelosis ovina. Arch Med Vet 27 ( $\mathrm{N}^{\circ}$ extraordinario), 113-117.

Arévalo S, B Otto, X Rojas. 2004. Determinación de brucelosis ovina (Brucella ovis), en predios de la Undécima Región de Chile. Resúmenes XIII Congreso Chileno de Medicina Veterinaria, Valdivia, Chile.

Blasco, J M. 1990. Brucella ovis. In: Nielsen K, Duncan J R (eds). Animal Brucellosis, CRC Press, Boca Raton, Florida, USA, Pp 351-378.

Brenner H, O Gefeller. 1997. Variation of sensitivity, specificity, likehood ratios and predictive values with disease prevalence. Stat Med 16, 981-991.

CHILE. 1996. Instituto Nacional de Estadísticas. VI Censo Nacional Agropecuario 1995 - 1996. XII Región de Magallanes y de la Antártica Chilena.

Estein S M. 1999. Aspectos inmunológicos en el diagnóstico y control de la Epididimitis contagiosa del carnero por Brucella ovis. Arch Med Vet 31, 5-17.

Gaffuri, A, C Garbarino. 1999. Piano regionale di controllo ed eradicazione della Brucella Ovis. L' Osservatorio, Revista bimestral de información científica del observatorio epidemiológico veterinario de la Región de Lombardía. Año N² 2, Abril 1999.

Greiner M, I A Gardner. 2000a. Epidemiologic issues in the validation of veterinary diagnostic tests. Prev Vet Med 45, 3-22.

Greiner M, D Pfeiffer, R D Smith. $2000^{\mathrm{b}}$. Principles and practical application of the receiver operating characteristic (ROC) analysis for diagnostic tests. Prev Vet Med 45, 23-41.

Jacobson R H. 1988. Validation of serological assays for diagnosis of infectious diseases. Rev sci tech Off int Epiz 17, 469-486.

Kott R W, G C Halver, B Firehammer, V M Thomas. 1988. Relationship between Brucella ovis semen culture and various semen and serology parameters. Theriogenology 29, 961-970.

Martin W B, I D Aitken. 2000. Disease of Sheep. Blackwell, London, UK, Pp 512.

Niilo I. 1984. Diagnosis of ovine Brucellosis. Can Vet J 25, 118-119.

Paolicchi F A, J Bartolomé, A Patitucci, C Solanet, C M Campero. 1992. Seguimiento clínico, serológico y bacteriológico en carneros naturalmente infectados con Brucella ovis. Rev Med Vet 73, 46-52.

Rahaley R S, S M Dennis, M S Smeltzer. 1983. Comparison of the enzymelinked immunosorbent assay and complement fixation test for detecting Brucella ovis antibodies en sheep. Vet Rec 113, 467-470.

Reichel M P, D J Baber, P W Armitage, D Lampard, R S Whitley, F Hilbink. 1994. Eradication of Brucella ovis from the Falkland Islands 1997-1993. Vet Rec 134, 595-597.

Rowland C, C Fuentes, J Gómez, J F Alvarez. 2003. Barrera sanitaria interna para el ingreso de ovinos a la XII Región de Magallanes y Antártica Chilena. Resúmenes $10^{\circ}$ Simposium Internacional de Epidemiología y Economía Veterinaria ISVEE X, Viña del Mar, Chile. ID 619.

Ruelas D, R Rosadio. 1999. Desarrollo y estandarización de una prueba ELISA indirecta para brucelosis ovina. Rev Inv Vet Perú 10 , 43-55.

Searson J E. 1982. Sensitivity and especificity of two microtite complement fixation test for the diagnosis Brucella ovis infections in rams. Aust Vet $J$ 58, 5. 
Vigliocco A M, P S Silva Paulo, J Mestre, G C Briones, G Dragi, M Tossi, K Nielsen. 1997. Development and validation of an indirect enzyme linked immunoassay for detection of ovine antibody to Brucella ovis. Vet Microbiol 54, 357-368.

Walter R L, B R Lea Master, J N Srellflug, E L Riberstein. 1985. Use of enzyme-liked immunosorbent assay for detection of antibodies to Brucella ovis in sheep: field trial. Am J Vet Res 8, 1642-1643.

Worthington RW, W Wedell, M E Penrose. 1984. A comparison of three serological tests for the diagnosis of Brucella ovis infection in rams. New Zeal Vet J 32, 58-60.
Zamora J, E Chahuan, M Polette, O Alonso, X Rojas, J Kruze, M Hervé. 1977. Brucella ovis y otros agentes etiológicos en epididimitis y orquitis infecciosa ovina. Arch Med Vet 9, 94-99.

Zweig M H, G Campbell. 1993. Receiver-operating characteristic (ROC) plots: a fundamental evaluation tool in clinical medicine. Clin Chem 39, 561-577. 
